\section{On One-Multiplier Implementations of FIR Lattice Structures}

ZINNUR DOĞANATA AND P. P. VAIDYANATHAN

\begin{abstract}
One-multiplier realizations for certain recently reported FIR lossless lattice structures are investigated. The multiplier extraction approach is used to show that there does not exist a real one-multiplier realization whereas it is possible to get complex one-multiplier realizations. This is unlike the situation in conventional linear-prediction FIR lattice structures, where real one-multiplier realizations are possible.
\end{abstract}

\section{INTRODUCTION}

Recently a procedure has been outlined for the synthesis of a cascaded lossless lattice structure that can realize any pair of power-complementary FIR transfer functions [1]. Each building block of the structure has four multipliers. However it is possible to obtain a denormalized structure which has only two multipliers per building block from the previous one, by proper scaling. The denormalized structure is shown in Fig. 1. Since it resembles the LPC lattice which has one-multiplier realizations, it is natural to ask if we can reduce the number of multipliers per building block to one. In this correspondence we use the multiplier extraction approach [2] to show that it is not possible to get structures that have one real multiplier per building block. This is unlike the situation in conventional linear-prediction FIR lattice structures, where real one-multiplier realizations are possible. The multiplier extraction approach also places in evidence a second derivation of the one-multiplier structure derived in [3], for the well-known FIR lattice [4].

\section{One-Multiplier Realizations for the CASCADED LOSSLESS LATTICE}

The denormalized structure of Fig. 1 is a cascade of building blocks separated by delays. The input-output equations corresponding to a building block are given by

$$
\left(\begin{array}{l}
y_{1} \\
y_{2}
\end{array}\right)=S\left(\begin{array}{l}
x_{1} \\
x_{2}
\end{array}\right)=\left(\begin{array}{ll}
s_{11} & s_{12} \\
s_{21} & s_{22}
\end{array}\right)\left(\begin{array}{l}
x_{1} \\
x_{2}
\end{array}\right)=s\left(\begin{array}{rr}
1 & -\alpha \\
\alpha & 1
\end{array}\right)\left(\begin{array}{l}
x_{1} \\
x_{2}
\end{array}\right)
$$

where $s$ is a nonzero but otherwise arbitrary scale factor. We wish to find a new building block that will realize (1) with only one multiplier $m$. We, therefore, consider the constrained threepair [5] shown in Fig. 2, that contains only adders and multiplierless connections. The input-output equations of the three-pair are given in matrix form as

$$
\left(\begin{array}{l}
y_{1} \\
y_{2} \\
y_{3}
\end{array}\right)=T\left(\begin{array}{l}
x_{1} \\
x_{2} \\
x_{3}
\end{array}\right)=\left(\begin{array}{lll}
t_{11} & t_{12} & t_{13} \\
t_{21} & t_{22} & t_{23} \\
t_{31} & t_{32} & t_{33}
\end{array}\right)\left(\begin{array}{c}
x_{1} \\
x_{2} \\
x_{3}
\end{array}\right)
$$

where $t_{i j}$ are the transfer parameters. Constraining $t_{33}$ to be zero

Manuscript received June 14, 1987. This work was supported in part by the National Science Foundation under Grant DCI 8552579 , in part by Caltech's programs in Advanced Technology grant sponsored by Aerojet General, General Motors, GTE, and TRW, and in part by the Naval Oceans Systems Center through a subcontract by the San Diego State University Foundation.

The authors are with the Department of Electrical Engineering, California Institute of Technology, Pasadena, CA 91125

IEEE Log Number 8717340.

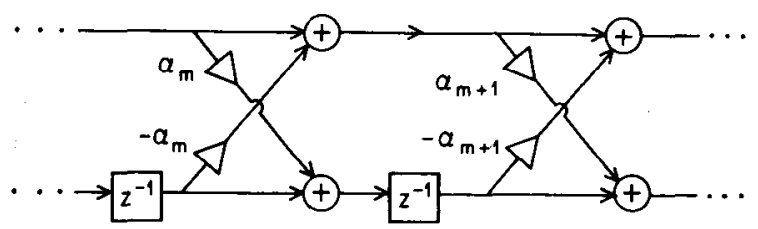

Fig. 1. The denormalized lattice structure.

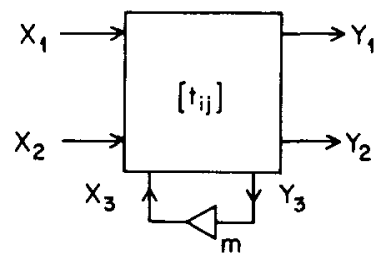

Fig. 2. The constrained three-pair.

in order to avoid a delay-free loop, we have

$$
\begin{aligned}
\left(\begin{array}{l}
y_{1} \\
y_{2}
\end{array}\right) & =\left(\begin{array}{ll}
t_{11} & t_{12} \\
t_{21} & t_{22}
\end{array}\right)\left(\begin{array}{l}
x_{1} \\
x_{2}
\end{array}\right)+\left(\begin{array}{l}
t_{13} \\
t_{23}
\end{array}\right) x_{3} \\
y_{3} & =\left(\begin{array}{ll}
t_{31} & t_{32}
\end{array}\right)\left(\begin{array}{l}
x_{1} \\
x_{2}
\end{array}\right) \\
x_{3} & =m y_{3}=m\left(\begin{array}{ll}
t_{31} & t_{32}
\end{array}\right)\left(\begin{array}{l}
x_{1} \\
x_{2}
\end{array}\right) .
\end{aligned}
$$

Substituting (3c) in (3a), we get

$$
\left(\begin{array}{l}
y_{1} \\
y_{2}
\end{array}\right)=\left(\begin{array}{ll}
t_{11}+m t_{13} t_{31} & t_{12}+m t_{13} t_{32} \\
t_{21}+m t_{23} t_{31} & t_{22}+m t_{23} t_{32}
\end{array}\right)\left(\begin{array}{c}
x_{1} \\
x_{2}
\end{array}\right)
$$

Comparing (4) with (1), we conclude that a one-multiplier realization must necessarily satisfy

$$
\begin{aligned}
& t_{11}+m t_{13} t_{31}=s \\
& t_{12}+m t_{13} t_{32}=-s \alpha \\
& t_{21}+m t_{23} t_{31}=s \alpha \\
& t_{22}+m t_{23} t_{32}=s .
\end{aligned}
$$

If there exist $m$ and $s$ such that (5a) to (5d) hold for an arbitrary real-valued $\alpha$ and a fixed matrix $T=\left[t_{i j}\right]$ independent of both $m$ and $\alpha$, the following equations must hold:

$$
\begin{aligned}
t_{11} & =t_{22} \\
t_{13} t_{31} & =t_{23} t_{32} \\
t_{12} & =-t_{21} \\
t_{13} t_{32} & =-t_{23} t_{31} .
\end{aligned}
$$

Using (6b) and (6d) we can write

$$
t_{31}^{2}+t_{32}^{2}=0 .
$$

If we restrict $t_{i j}$ to be real, (7) implies that $t_{31}$ and $t_{32}$ are both zero and thus (5a) to (5d) become

$$
t_{11}=s \quad t_{12}=-s \alpha \quad t_{21}=s \alpha \quad t_{22}=s
$$

which implies that $t_{11} / t_{12}=-1 / \alpha$. Therefore, there does not exist a real one-multiplier realization for the cascaded lossless lattice.

If we permit $t_{i j}$ to be complex, then (6a)-(6d) can be satisfied. For example, we can choose $t_{13}=t_{32}=1, t_{23}=t_{31}=j$ to satisfy 


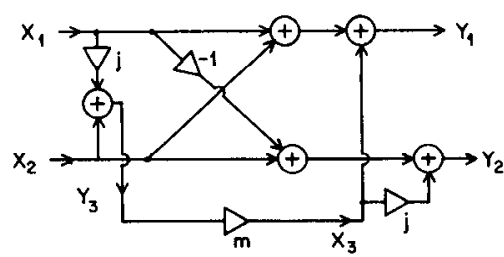

Fig. 3. A complex one-multiplier realization.

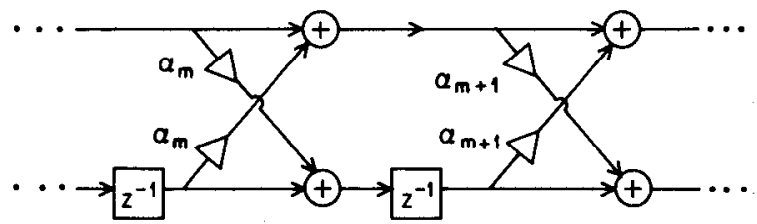

Fig. 4. The LPC lattice.

(6b),(6d) and choose $t_{11}=t_{12}=-t_{21}=t_{22}=1$ to satisfy (6a), (6c). With this choice of $T=\left[t_{i j}\right]$, we have

$$
s=1+j m, \quad \alpha=-(1+m) /(1+j m)
$$

or equivalently, in terms of $\alpha$

$$
s=(1-j) /(1+j \alpha) \quad m=-(1+\alpha) /(1+j \alpha) .
$$

The resulting structure is shown in Fig. 3.

\section{ONE-MULTIPLIER REALIZATIONS FOR THE FIR LPC LATTICE}

A well-known FIR lattice structure is the one that arises in the context of linear predictive coding [4]. As shown in Fig. 4, this structure has two multipliers in each building block. The input-output equations corresponding to a building block are given as

$$
\left(\begin{array}{l}
y_{1} \\
y_{2}
\end{array}\right)=S\left(\begin{array}{l}
x_{1} \\
x_{2}
\end{array}\right)=\left(\begin{array}{ll}
s_{11} & s_{12} \\
s_{21} & s_{22}
\end{array}\right)\left(\begin{array}{l}
x_{1} \\
x_{2}
\end{array}\right)=s\left(\begin{array}{ll}
1 & \alpha \\
\alpha & 1
\end{array}\right)\left(\begin{array}{l}
x_{1} \\
x_{2}
\end{array}\right) .
$$

It is known that other lattice forms can be derived based on this one. Makhoul [3] has derived some intermediate lattice structures from which he obtains one-multiplier structures that realize (11). In the following, the same one-multiplier realization is derived directly using the multiplier extraction approach.

Comparing (4) and (11), we write

$$
\begin{array}{ll}
t_{11}+m t_{13} t_{31}=s & t_{12}+m t_{13} t_{32}=s \alpha \\
t_{21}+m t_{23} t_{31}=s \alpha & t_{22}+m t_{23} t_{32}=s .
\end{array}
$$

If we choose

$$
t_{22}=t_{11}=1 \quad t_{12}=t_{21}=0
$$

equation (12) becomes

$$
1+m t_{13} t_{31}=s \quad m t_{13} t_{32}=s \alpha \quad m t_{23} t_{31}=s \alpha \quad 1+m t_{23} t_{32}=s .
$$

With $t_{13}=t_{31}=t_{23}=t_{32}=1,(14)$ is satisfied and we get

$$
s=\frac{1}{1-\alpha} \quad m=\frac{\alpha}{1-\alpha} .
$$

The corresponding structure is shown in Fig. 5. The other one-

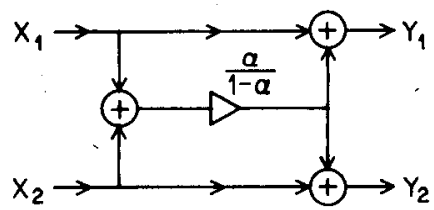

Fig. 5. One-multiplier realization for the LPC lattice.

multiplier structures in [3] can similarly be obtained by appropriate choices of $\boldsymbol{T}=\left[t_{i j}\right]$.

\section{REFERENCES}

[1] P. P. Vaidyanathan, "Passive cascaded lattice structures for low-sensitivity FIR filter design," IEEE Trans. Circuits Syst., vol. CAS-33, pp. 1045-1064, Nov. 1986.

[2] S. K. Mitra and R. J. Sherwood, "Digital ladder networks," IEEE Trans. Audio Electroacoust., vol. AU-21, pp. 30-36, Feb. 1973.

[3] J. Makhoul, "A class of all-zero lattice digital filters: Properties and applications," IEEE Trans. Acoust., Speech, Signal Processing, vol. ASSP-26, pp. 304-314, Aug. 1978.

[4] F. Itakura and S. Saito, "Digital filtering techniques for speech analysis and synthesis," in Proc. 7th Int. Cong. Acoust., Budapest, Hungary, pp. 261-264, 1971.

[5] S. K. Mitra and K. Hirano, "Digital allpass networks," IEEE Trans Circuits Syst., vol. CAS-21, pp. 688-700, Sept. 1974.

\section{An Efficient Technique to Improve NORA CMOS Testing}

\author{
NAM LING AND MAGDY A. BAYOUMI
}

\begin{abstract}
This paper presents a novel circuit technique to improve the testability of NORA (NO RAce) CMOS circuits. It is based on the structure, properties and operations of NORA CMOS. The precharge and evaluation properties of NORA CMOS enable one to design simple testing circuit for output stuck-at-zero, stuck-at-one, stuck-open and stuck-on faults. Area and time considerations, as well as the applications of this testability enhancement technique are also discussed.
\end{abstract}

\section{INTRODUCTION}

With the increase in the complexity of VLSI systems, testing has become more difficult and this has been the motivation for adopting "design for testability" strategy in which several design techniques are used to make testing manageable and economical. An example of such methods is the built-in-self-test (BIST) technique, which has been introduced to achieve more economical and effective testing strategy [6]. In this approach, the implementation technology has a great impact on the testing technique to be used which has necessitated developing special methods for each technology. Testing NORA (NO RAce) CMOS [1], [2] is the main scope of this paper.

NORA is a racefree dynamic CMOS for pipelined logic structures [2]. It operates using two clocks in racefree fashion regardless of their overlap time and clock skew. The importance of NORA circuits stems from this property which overcome several problems of Domino CMOS circuits [2].

The main building block of NORA technique is shown in Fig. 1. Two clocks $\phi$ and $\bar{\phi}$ are provided. During the precharge phase

Manuscript received May 7, 1987; revised August 7, 1987.

The authors are with the Center of Advanced Computer Studies, University of Southwestern Lousiana, Lafayaette, LA 70504.

IEEE Log Number 8717342. 